\title{
Monomeric type I and type III transforming growth factor- $\beta$ receptors and their dimerization revealed by single-molecule imaging
}

\author{
Wei Zhang ${ }^{1}$, Jinghe Yuan ${ }^{1}$, Yong Yang ${ }^{1}$, Li Xu ${ }^{1}$, Qiang Wang ${ }^{2,3}$, Wei Zuo ${ }^{2}$, Xiaohong Fang ${ }^{1}$, Ye-Guang Chen ${ }^{2}$
}

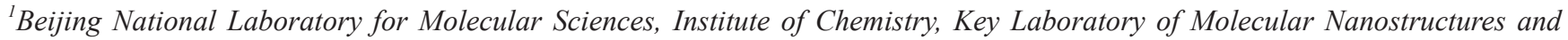
Nanotechnology, Chinese Academy of Sciences, Beijing 100190, China; ${ }^{2}$ State Key Laboratory of Biomembrane and Membrane Biotechnology, School of Life Sciences, Tsinghua University, Beijing 100084, China

Transforming growth factor- $\beta$ (TGF- $\beta$ ) binds with two transmembrane serine/threonine kinase receptors, type

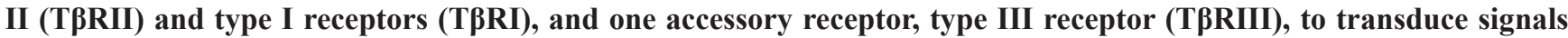

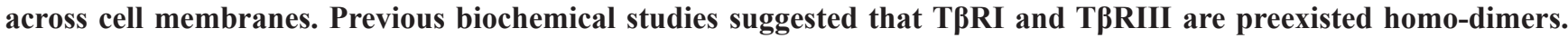
Using single-molecule microscopy to image green fluorescent protein-labeled membrane proteins, for the first time we have demonstrated that T $\beta R I$ and T $\beta R I I I$ could exist as monomers at a low expression level. Upon TGF- $\beta 1$ stimu-

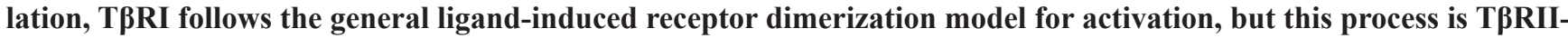

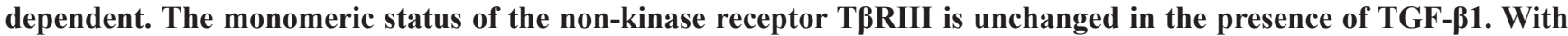
the increase of receptor expression, both T $\beta R I$ and T $\beta R I I I$ can be assembled into dimers on cell surfaces.

Keywords: single-molecule fluorescence; TGF- $\beta$ signaling; Type I TGF- $\beta$ receptor; Type III TGF- $\beta$ receptor; subunit stoichiometry

Cell Research (2010) 20:1216-1223. doi:10.1038/cr.2010.105; published online 13 July 2010

\section{Introduction}

Transforming growth factor- $\beta$ (TGF- $\beta$ ) and related growth factors regulate a diverse range of important cellular functions, including cell proliferation, differentiation, motility, and apoptosis [1-4]. Three cell-surface

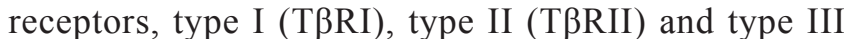
(T $\beta$ RIII) receptors, are involved in TGF- $\beta$ signal transduction. T $\beta R I$ and T $\beta$ RII receptors are glycoproteins with estimated molecular weights of 53 and $73 \mathrm{kDa}$. They belong to the serine/threonine kinase family and are essential to transduce TGF- $\beta$ signal. T $\beta$ RIII or betaglycan, the most abundant TGF- $\beta$ receptor subtype in many cell

Correspondence: Xiaohong Fang, ${ }^{\mathrm{a}}$ Ye-Guang Chen

aE-mail: xfang@iccas.ac.cn

${ }^{\mathrm{b}}$ E-mail: ygchen@mail.tsinghua.edu.cn

${ }^{3}$ Current address: Institute of Zoology, Chinese Academy of Sciences, Beijing 100101, China.

Received 11 April 2010; revised 11 May 2010; accepted 24 May 2010; published online 13 July 2010 types, is a large proteoglycan (approximately $280 \mathrm{kDa}$ ). It is a non-kinase receptor without a signaling motif, but regulates TGF- $\beta$ 's access to the signaling receptors.

TGF- $\beta$ signaling is triggered by the ligand-receptor binding [1]. A ligand, such as TGF- $\beta 1$, first binds to its specific receptor T $\beta R I I$, allowing the subsequent recruitment of T $\beta R I$ to form a heteromeric signaling complex of T $\beta R I / T \beta R I I$. This leads to the phosphorylation of T $\beta R I$ by T $\beta R I I$ and then the phosphorylation of Smad proteins by T $\beta$ RI to propagate the signal to the cell nucleus. The accessory receptor T $\beta$ RIII promotes TGF- $\beta$ signaling by increasing ligand accessibility to the signaling complex T $\beta R I / T \beta R I I$. Study of the stoichiometry of TGF- $\beta$ receptors and their oligomerization status before and after ligand binding is of critical importance to understand the molecular nature of TGF- $\beta$ signaling complex formation during signal transduction. According to the proposed TGF- $\beta$ signaling model, all the three TGF- $\beta$ receptors are believed to exist as dimers or oligomers in the resting cells [5-7]. Upon ligand stimulation, TGF- $\beta$ recruits two homo-dimers of T $\beta R I$ and T $\beta R I I$ to form 
the signaling complex [8]. The heteromeric complexes from T $\beta$ RIII dimers and T $\beta$ RII dimers are minor and transient species during the signal process. This model suggests that the activation of TGF- $\beta$ receptors is different from that of tyrosine kinase receptors, another major class of membrane receptor protein kinases. It has been well documented that the tyrosine kinase receptors exist as monomers in resting cells and follow the general rule of ligand-induced dimerization for activation. As previous investigations on TGF- $\beta$ receptor signaling complex were mainly based on in vitro biochemical assays with overexpressed proteins or cell lysates, such as double immunoprecipitation, sedimentation velocity analysis, and antibody-mediated immunofluorescence co-patching, the results may not represent the molecular nature under physiological conditions [9].

Recent advances in single-molecule fluorescence imaging have offered a new way to analyze membrane proteins with ultrasensitivity and probe their stoichiometry in intact cells [10-14]. Using green fluorescent protein (GFP) to label the membrane proteins, stepwise photobleaching curves of individual proteins are counted, and a binomial distribution of photobleaching steps is obtained to investigate protein stoichiometry. With the single-molecule imaging technique, we have investigated the stoichiometry and activation mechanism of the primary TGF- $\beta$-binding receptor T $\beta$ RII [13]. For the first time, we observed that T $\beta$ RII mainly exists as monomer at a low expression level and dimerizes upon ligand stimulation. We thus expected that the serine-threonine kinase receptors can be activated in the same way as tyrosine kinase receptors [15].

To further investigate whether the mode of receptor activation via dimerization of monomers can be generalized to the serine/threonine kinase receptors and whether other TGF $\beta$ receptors have their characteristic oligomerization structures, we expanded our study to the other two receptors involved in signaling, T $\beta R I$ and T $\beta R I I I$ receptors, with single-molecule microscopy. Here, we showed that T $\beta R I$ and T $\beta R I I I$ also existed as monomers at low expression. After TGF- $\beta 1$ stimulation, T $\beta R I$ could dimerize when T $\beta$ RII was coexpressed, while T $\beta$ RIII remained monomeric. Therefore, the model of ligand-induced dimerization could be extended to the two kinase receptors, T $\beta R I$ and T $\beta$ RII, in TGF- $\beta$ signal transduction, but not the accessory receptor, T $\beta$ RIII. With the increase of the receptor density on cell surfaces, monomeric T $\beta \mathrm{RI}$ and T $\beta$ RIII receptors were assembled into dimers spon-

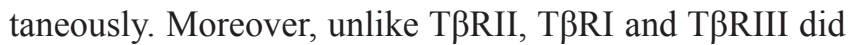
not aggregate into higher-order clusters when they were highly expressed. Our results not only support the recently proposed new activation model of TGF $\beta$ receptors, but also provide new information on the aggregation status of T $\beta R I$ and T $\beta R I I I$ for better understanding of TGF $\beta$ receptor complex formation and their signaling processes.

\section{Results}

Monomeric TBRI in the resting cells at a low receptor density

To investigate the stoichiometry of T $\beta$ RI receptors, we tagged T $\beta R I$ at its $\mathrm{C}$-terminus with enhanced GFP. The T $\beta R I-G F P$ was functional in activating the expression of the TGF- $\beta$-responsive reporter CAGA-luciferase in the presence of TGF- $\beta$ [16] (Supplementary information, Figure S1). Single-molecule fluorescence imaging of the transfected T $\beta$ RI-GFP was examined in three different types of cells, HeLa, MCF7 and R1B/L17 cells, which have different amounts of endogenous T $\beta R I$. MCF7, which has undetectable expression of T $\beta R I$ and T $\beta R I I$ [17], and L17, which has undetectable expression of T $\beta R I$ [18], were used to exclude the influence of endogenous receptors and study whether TGF- $\beta$-induced T $\beta$ RI dimerization is T $\beta$ RII-dependent.

We first studied T $\beta R I$ expression on the resting HeLa cells using an approach similar to that used for T $\beta$ RII earlier [13]. A small amount of plasmids $(0.25 \mu \mathrm{g})$ encoding T $\beta$ RI-GFP was transfected into the cells. The cells were fixed and imaged at $4 \mathrm{~h}$ after transfection. This ensured that T $\beta$ RI-GFP were expressed at a low density (less than 50 molecules in a $15 \times 15 \mu \mathrm{m}^{2}$ area) to distinguish individual T $\beta R I-G F P$ under fluorescence microscopy. As shown in the typical total internal reflection fluorescence microscopy (TIRFM) image (Figure 1A), most T $\beta R I-G F P$ molecules appeared as well-dispersed diffraction-limited fluorescent spots $(5 \times 5$ pixels, $800 \times$ $800 \mathrm{~nm}$ ), and maintained their fluorescence mostly for less than $5 \mathrm{~s}$ before photobleaching.

To investigate whether these diffraction-limited spots represented monomeric T $\beta R I$ tagged with one GFP molecule, we counted the photobleaching steps of individual fluorescent T $\beta$ RI-GFP molecules. It has been demonstrated that the statistical analysis of bleaching steps of GFP-fused membrane proteins is a new way to determine the subunit stoichiometry of membranebound proteins $[10,11]$. From the distribution of the photobleaching steps of T $\beta R I-G F P$ spots on HeLa cell membranes (from 13 cells), we found $82.1 \%$ (487 of 589 spots) bleached in one step, and 16.1\% (92 of 589) bleached in two steps (Figure 1B, 1C and Supplementary information, Figure S2). This result was similar to what we obtained for T $\beta$ RII, where the majority of the receptors had the one-step photobleaching characteristics [13], indicating that they were monomers instead of dimers. Besides, the intensity distribution of T $\beta$ RI-GFP fluores- 
A

B
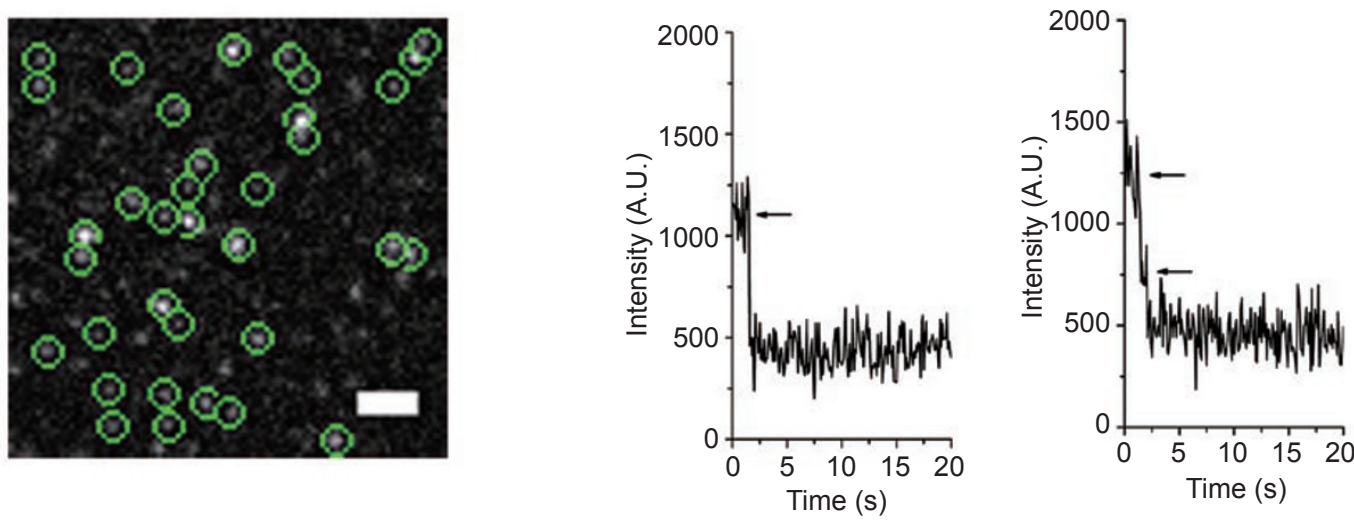

C
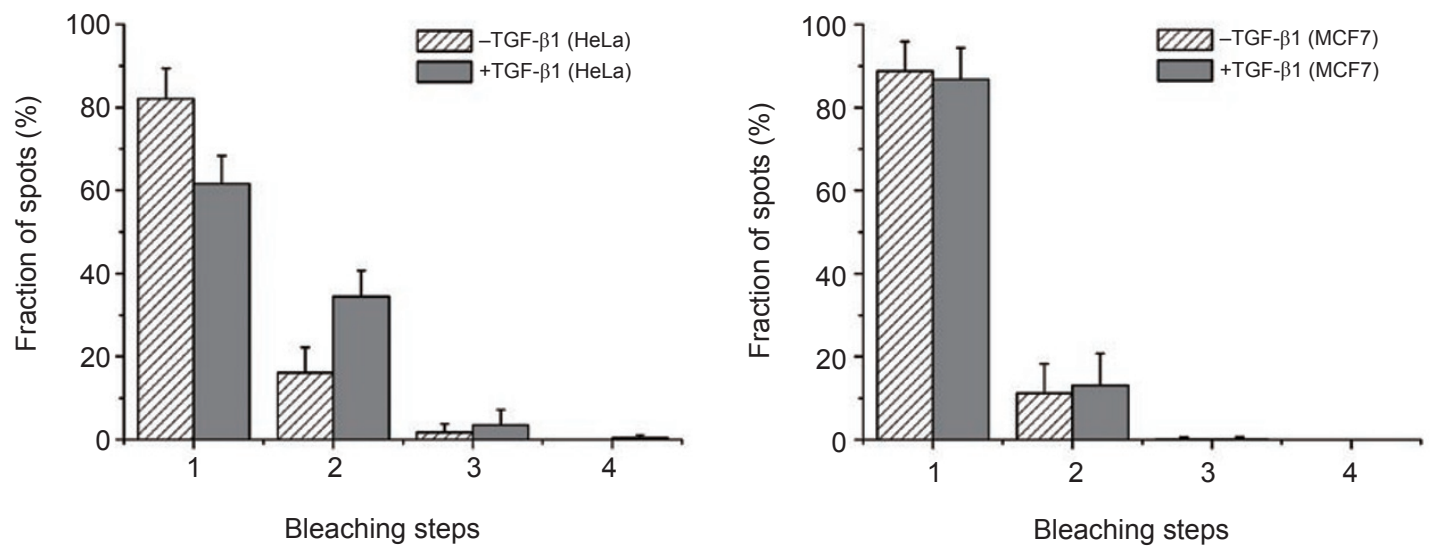

Figure 1 Single-molecule fluorescence imaging of T $\beta R$ I molecules. (A) A typical single-molecule image of T $\beta R I-G F P$ on the resting HeLa cell membrane. The image was averaged from the first five frames of a raw movie. The diffraction-limited spots $(5$ $\times 5$ pixel regions) enclosed with green circles represented the signals from individual T $\beta R I-G F P$ molecules. Scale bar: $2 \mu \mathrm{m}$. (B) Two representative time courses of GFP emission after background correction show one- and two-step bleaching. Arrows indicate the bleaching steps. (C, D) Frequency of one-step and multistep bleaching events for T $\beta$ RI-GFP before (dashed bar) and after (grey bar) TGF- $\beta 1$ stimulation in HeLa (C) and MCF7 cells (D), respectively. There is a significant statistical difference in the fraction of one- and two- step photobleaching for (C) $(P<0.001$ in the Student's $t$-test), but no significant difference for (D) $(P>0.05)$.

cent spots and their photobleaching time were also close to those of GFP-labeled EGFR molecules which were known as monomers on the HeLa cell membranes (data not shown), confirming that T $\beta$ RI-GFP molecules mainly existed as monomers.

As HeLa cells had moderate endogenous T $\beta$ RI, we then imaged T $\beta$ RI-GFP with MCF7 and R1B/L17 cells which have undetectable T $\beta R I$ expression to exclude the influence of endogenous receptors [17-19]. The results showed that $88.7 \%$ (265 of 303 spots from 10 cells) of the receptors on the membrane of MCF7 cells, and $89.3 \%$ ( 236 of 267 spots from 11 cells) of the receptors on the membrane of R1B/L17 cells, bleached in one step (Figure 1D and Supplementary information, Figure S3), consistent with that from HeLa cells. This dominance of one-step bleaching indicated that T $\beta R I$ indeed existed as monomers and endogenous receptors had no influence on the stoichiometry analysis of T $\beta$ RI-GFP.

TRRII-dependent dimerization of monomeric TRRI after $T G F-\beta 1$ stimulation

TGF- $\beta$ and related factors signal by binding to and bringing together pairs of type I and II receptors [20]. As our previous study demonstrated that monomeric T $\beta$ RIIGFP molecules dimerized upon TGF- $\beta 1$ stimulation [13], we asked whether the monomeric T $\beta R I$ also underwent dimerization in the presence of ligands.

HeLa cells which had a moderate level of T $\beta$ RII ex- 
pression were stimulated with TGF- $\beta 1$ at $4 \mathrm{~h}$ after T $\beta$ RIGFP transfection and were kept at $4{ }^{\circ} \mathrm{C}$ for $15 \mathrm{~min}$ to avoid receptor internalization. Then cells were fixed and imaged by TIRFM. According to the statistical analysis of the distribution of bleaching steps, TGF- $\beta 1$ treatment resulted in a significant decrease of monomeric T $\beta R I$ (from $82.1 \%$ to $61.6 \%$ ) and a corresponding increase of dimeric T $\beta$ RI, which bleached in two steps (from 16.1\% to $34.4 \%$ ) (Figure 1C).

Then we tested T $\beta$ RI activation in L17 cells which were deficient of endogenous T $\beta R I$ but expressed T $\beta R I I$ [18]. Compared to the cells without TGF- $\beta 1$ stimulation, in which $89.3 \%$ of fluorescent spots bleached in one step and $10.5 \%$ bleached in two steps, the population of the one-step bleaching spots decreased to $75.4 \%$, but that of the two-step bleaching spots increased to $22.1 \%$ (Supple- mentary information, Figure S3). The results implicated that TGF- $\beta 1$ could induce T $\beta$ RI dimerization in the presence of T $\beta$ RII.

To support this notion, we performed the imaging and analysis with MCF7 cells which were deficient of both endogenous T $\beta R I$ and T $\beta$ RII [17], and found that the distribution of photobleaching steps in the stimulated cells was almost the same as that of unstimulated cells: $86.8 \%$ of fluorescent spots from the TGF- $\beta 1$-treated cells bleached in one step, and $13.1 \%$ bleached in two steps (Figure 1D). These data together indicated that TGF$\beta$-induced T $\beta$ RI dimerization was T $\beta$ RII-dependent. Our results from single-molecule imaging of T $\beta R I-G F P$ were in agreement with the reported signaling model that TGF- $\beta 1$ does not bind to T $\beta R I$ without T $\beta R I I$, and that the TGF- $\beta 1 / T \beta R I I$ complex recruits T $\beta R I$ to form a
A

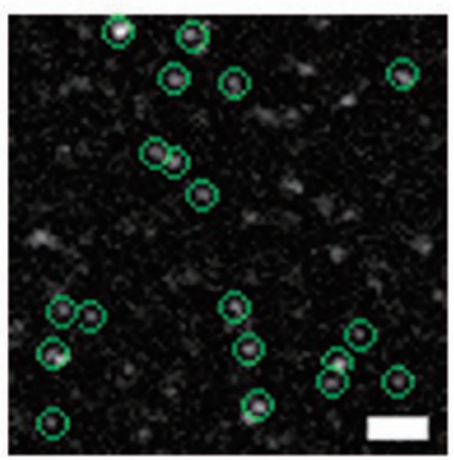

C

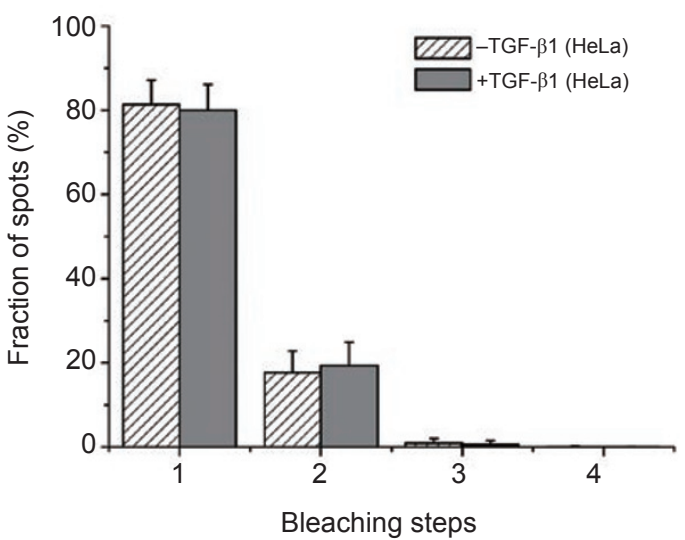

B
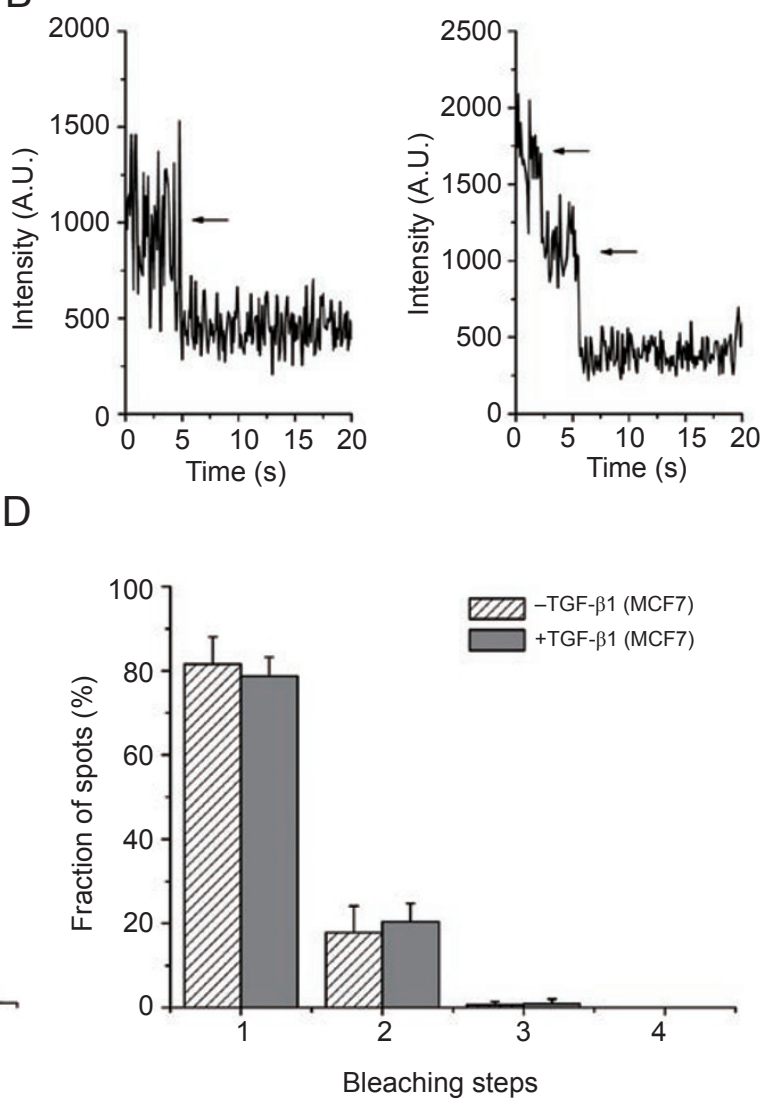

Figure 2 Single-molecule fluorescence imaging of T $\beta$ RIII molecules. (A) A typical single-molecule image of T $\beta$ RIII-GFP on the resting HeLa cell membrane. The image was averaged from the first five frames of a raw movie. The diffraction-limited spots (5 $\times 5$ pixel regions) enclosed with green circles represented the signals from individual T $\beta$ RIII-GFP molecules. Scale bar: $2 \mu \mathrm{m}$. (B) Two representative time courses of GFP emission after background correction show one- and two-step bleaching. Arrows indicate the bleaching steps. (C, D) Frequency of one-step and multistep bleaching events for T $\beta$ RIII-GFP before (dashed bar) and after TGF- $\beta 1$ stimulation (grey bar) in HeLa (C) and MCF7 (D) cells, respectively. There is no significant statistical difference in the fraction of one- and two-step photobleaching for either (C) or (D) $(P>0.05$ in the Student's $t$-test). 
ligand-receptor complex containing one dimeric ligand, two T $\beta$ RII and two T $\beta R I$ molecules $[20,21]$.

Monomeric TBRIII in the resting cells at a low receptor density

Using a similar approach, we investigated the stoichi-

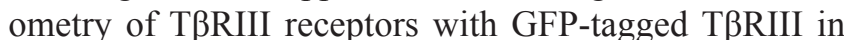
the three cell types, HeLa cells with endogenous T $\beta$ RIII, MCF7 and L6 cells which had few endogenous TRRIII receptors $[17,22]$.

Single-molecule fluorescence imaging of T $\beta$ RIII-GFP also showed that the majority of T $\beta$ RIII-GFP molecules $(81.3 \%$ in HeLa cells, $81.5 \%$ in MCF7 cells, $77.58 \%$ in L6 cells) photobleached in one step (Figure 2 and Supplementary information, Figure S4). Moreover, the intensity distribution of T $\beta$ RIII-GFP fluorescent spots and their photobleaching life times were all similar to those of single GFP-labeled EGFR molecules. Therefore, T $\beta$ RIII also existed as a monomer instead of a dimer at a low density.

\section{TBRIII remained monomeric after TGF- $\beta 1$ stimulation}

T $\beta$ RIII is involved in TGF- $\beta 1$ signaling together with T $\beta R I$ and T $\beta R I I$, but it does not belong to serine/threonine kinase receptors. Then we asked whether it follows ligand-induced dimerization.

HeLa cells, which had moderate T $\beta R I I$ and T $\beta R I$ and abundant T $\beta$ RIII expression, were used to study the oligomeric status of T $\beta$ RIII after ligand stimulation. At $4 \mathrm{~h}$ post-transfection with T $\beta$ RIII-GFP, the cells were treated with TGF- $\beta 1$ at $4{ }^{\circ} \mathrm{C}$ for $15 \mathrm{~min}$. Then cells were fixed and imaged by TIRFM. According to the bleaching-step analysis, no significant change was found before and after stimulation in the distributions of one-step bleaching molecules $(81.3 \%$ vs $80.0 \%)$ and two-step bleaching molecules (17.7\% vs $19.4 \%$ ) (Figure 2$)$. The results suggest that in the presence of T $\beta$ RII and T $\beta$ RI, TGF- $\beta 1$ could not induce TRRIII dimerization.

A
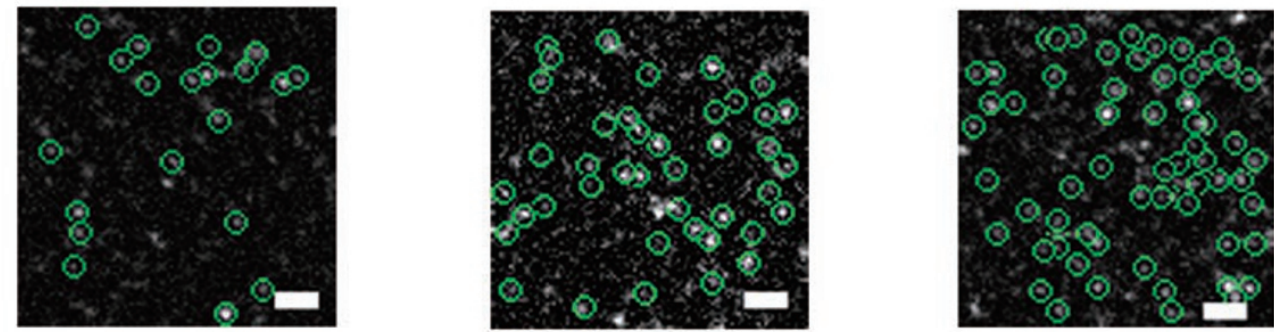

B

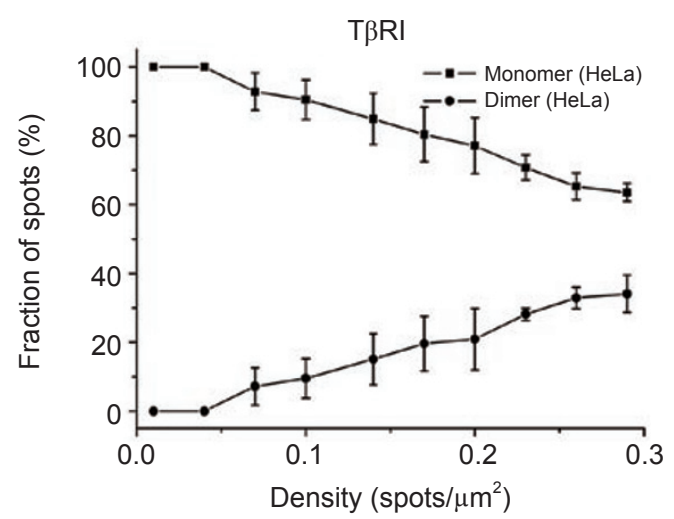

C

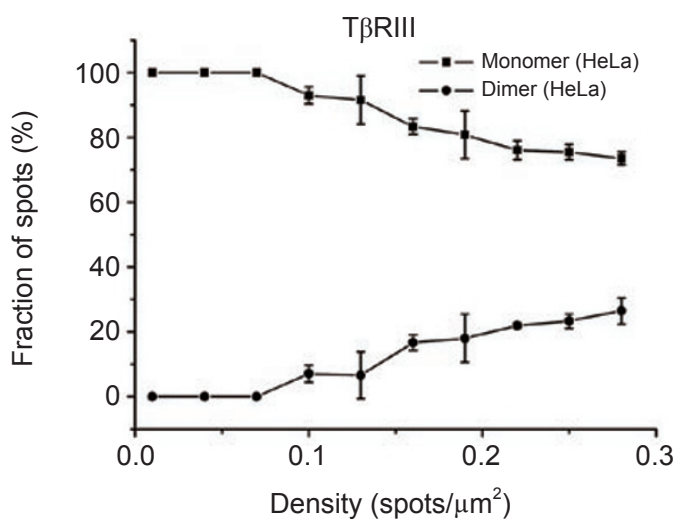

Figure $3 \mathrm{~T} \beta \mathrm{RI}$ and T $\beta \mathrm{R} I \mathrm{II}$ receptors were assembled into dimers as their concentrations on HeLa cell membranes increased. (A) Typical single-molecule images of GFP-labeled T $\beta R$ I at different densities (left: $0.1 \mathrm{spots} / \mu \mathrm{m}^{2}$, middle: $0.2 \mathrm{spots} / \mu \mathrm{m}^{2}$, right: $0.3 \mathrm{spots} /$ $\left.\mu \mathrm{m}^{2}\right)$ on HeLa cell membranes. Each image was averaged from the first five frames of a raw movie. The diffraction-limited spots $(5 \times$ 5 pixel regions) enclosed with green circles represented the signals from individual GFP-labeled receptors. Image area: $15 \times 15 \mu \mathrm{m}^{2}$. Scale bar: $2 \mu \mathrm{m}$. (B) The change in the fraction of monomer and dimer with the density of T $\beta$ RI-GFP fluorescent spots in HeLa cells. (C) The change in the fraction of monomer and dimer with the density of T $\beta R$ III-GFP fluorescent spots in HeLa cells. Different receptor densities were calculated from the cells having different receptor expression levels. Each ratio was averaged from the data obtained from three cells. 
We further investigated MCF7 cells which had few endogenous T $\beta R I, T \beta R I I$ and T $\beta$ RIII receptors, as well as L6 cells which had moderate T $\beta R I$ and T $\beta R I I$ expression but no T $\beta R I I I[17,22]$. The fractions of either onestep or two-step bleaching fluorescent spots of T $\beta$ RIIIGFP showed no obvious change after TGF- $\beta 1$ stimulation (Figure 2 and Supplementary information, Figure S4). The results indicated that TGF- $\beta 1$ could not induce T $\beta$ RIII dimerization, which was different from that of

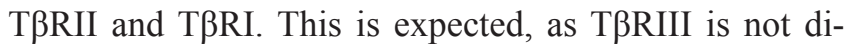
rectly involved in ligand-receptor complex formation for TGF- $\beta$ signaling. Our results suggest that monomeric T $\beta$ RIII may be competent enough to serve as an accessory receptor for TGF- $\beta 1$ binding with T $\beta$ RII.

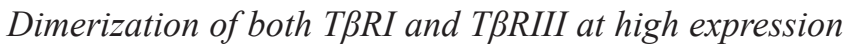 levels}

Our results demonstrated that, similar to T $\beta R I I, T \beta R I$ and T $\beta$ RIII exist in the monomeric form in the absence of ligand. This is in contrast to the previous reported model that T $\beta R I$ and T $\beta$ RIII are preformed homodimers. The possible reason is that previous reports on homodimeric receptors were based on conventional biochemical methods under high protein expression levels [13].

Therefore, we investigated the status of T $\beta R I-G F P$ and T $\beta$ RIII-GFP at higher expression levels by increasing the plasmid DNA concentration (from 0.25 to $0.5 \mu \mathrm{g}$ ) and expression time (from 4 to $8 \mathrm{~h}$ ). With the increased expression, more individual fluorescent spots were observed for both T $\beta R I$ and T $\beta$ RIII. They distributed almost homogenously as diffraction-limited spots with different densities at different expression levels. This was different from what was observed for TRRII. When highly ex- pressed, many T $\beta$ RII formed clusters which were larger and brighter than the diffraction-limited single-molecule spots [13]. If we further increased the expression using a transfection time of 8-16 h, the fluorescence signals for both T $\beta R I$ and T $\beta$ RIII were still uniform with few clusters (Supplementary information, Figure S5). However, no individual fluorescent spots could be differentiated under the spatial resolution of fluorescence microscopy, as the receptor density was too high.

We calculated the monomer-dimer ratio for the two receptors under different expression densities based on the assumption that the receptors were distributed homogenously throughout the plasma membrane. For each cell, an area of $15 \times 15 \mu \mathrm{m}^{2}$ was extracted and the distribution of bleaching steps for the spots was counted. We found that the fraction of dimer (with two bleaching steps) increased with the increase in density of T $\beta R I$ receptors on the cell membranes (Figures 3 and 4A). Taking T $\beta R I$ in HeLa cells as an example, when the density of spots was below 0.1 spots $/ \mu \mathrm{m}^{2}$, more than $90 \%$ of spots bleached in one step, indicating that T $\beta R I$ mainly existed as a monomer (Figure 3A). When the density of spots increased, the fraction of monomer decreased, while that of dimer increased, indicating that monomeric T $\beta R I$ molecules were spontaneously assembled into dimers. The above results suggested the possibility of monomer-dimer equilibrium on cell membranes for T $\beta R I$.

For T $\beta$ RIII receptors, the monomer-dimer ratio change was almost the same as T $\beta R I$ (Figures 3 and 4B). When the density of the spots was below $0.1 \mathrm{spots} / \mu \mathrm{m}^{2}$, T $\beta R I I I$ mainly existed as monomers. The ratio of monomer and dimer kept decreasing as the density of T $\beta$ RIII receptors increased. Thus, the monomer-dimer equilibrium on cell
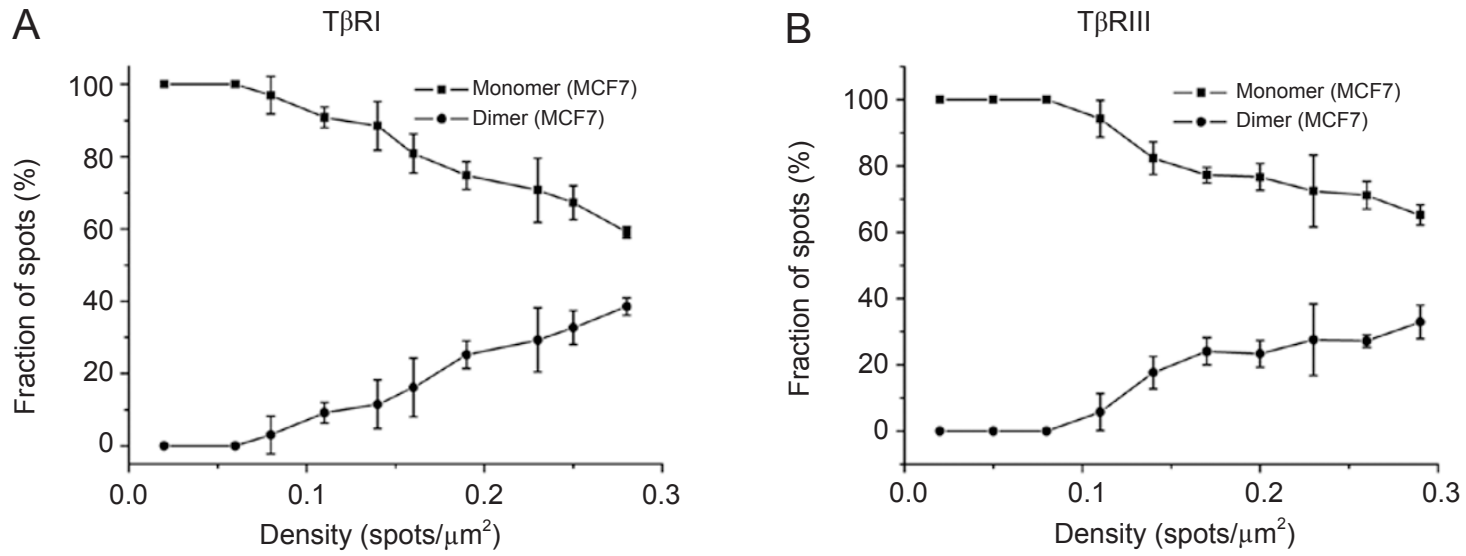

Figure $4 \mathrm{~T} \beta \mathrm{R}$ I and T $\beta$ RIII receptors were assembled into dimers as their expression on MCF7 cell membranes increased. (A) The change in the fraction of monomer and dimer with the density of TRRI-GFP fluorescent spots. (B) The change in the fraction of monomer and dimer with the density of T $\beta$ RIII-GFP fluorescent spots. Different receptor densities were calculated from the cells having different receptor expression levels. Each ratio was averaged from the data obtained from three cells. 
membranes might also exist for T $\beta$ RIII. We did not count the monomer-dimer ratio when the receptor density was greater than 0.3 spots $/ \mu \mathrm{m}^{2}$. Under the conditions with a higher protein density, the counting may not be accurate as more than $50 \%$ of the spots in the images did not show discrete one or two photobleaching steps and were discarded.

\section{Discussion}

For cell signaling by EGFR family members (EGFR/ ErbB1, ErbB2, ErbB3, ErbB4) of receptor tyrosine kinases, there is a general scheme for the role of receptor homo- and hetero-oligomerization in activation [23]. In the absence of ligands, inactive receptor monomers are in equilibrium with inactive or active receptor dimers. Ligand binding stabilizes the active dimer and accelerates protein tyrosine kinase activation.

In our study, we found that the serine/threonine kinase TGF- $\beta$ receptor monomers were also in equilibrium with receptor dimers. Upon ligand stimulation, while T $\beta$ RII underwent T $\beta$ RI-independent dimerization, the dimerization of T $\beta R I$ was T $\beta$ RII-dependent. Although it is possible that GFP labeling may interfere with the receptor activation and dimerization to some extent, the similar monomer-dimer population change before and after ligand stimulation for the GFP-labeled EGFR and the GFP-labeled TGF $\beta$ receptor (T $\beta R I$ and T $\beta R I I)$ suggests the similar activation model for the two types of receptors. Thus, we propose that TGF- $\beta$ binding induces the formation of activated T $\beta R I I$ homodimers, and then T $\beta R I$ dimerizes via its interaction with T $\beta$ RII dimers to form T $\beta R I I-T \beta R I$ heterotetramers, leading to the activation of T $\beta$ RI kinase. This could be further investigated by future colocalization or fluorescence energy transfer imaging of individual T $\beta R I I$ and T $\beta R I$ molecules labeled with different fluorescence proteins.

In conclusion, we have applied single-molecule TIRF microscopy to demonstrate the monomeric states of T $\beta$ RI and T $\beta$ RIII. The results reveal that monomeric and dimeric forms of both T $\beta R I$ and T $\beta R I I I$ exist on cell surfaces, with the majority being monomers in the resting cells at low expression levels. TRRI follows the ligand-induced dimerization model in a T $\beta$ RII-dependent manner, while the non-kinase receptor T $\beta R I I I$ does not dimerize upon TGF- $\beta$ stimulation. As receptor homodimerization and hetero-oligomerization have a critical role in TGF- $\beta$ signal transduction and we also observed a small portion of T $\beta$ RI molecules existing as dimers in the absence of TGF- $\beta$, further investigation is needed to examine whether the dynamic monomer-dimer distribution of the receptors plays a role in TGF- $\beta$ signaling in the absence of ligand.

\section{Materials and Methods}

\section{Plasmid construction}

The DNA fragments encoding full-length T $\beta$ RI and EGFR were subcloned into the HindIII and BamHI sites of pEGFP-N1 (Clontech), yielding the T $\beta$ RI-GFP and EGFR-GFP expression plasmids. The plasmid encoding HA-T $\beta$ RIII was a gift from Dr Gerard C Blobe (Duke University, USA). The DNA fragments encoding full-length HA-T $\beta$ RIII were subcloned into the HindIII and NheI sites of pEGFP-N1 (Clontech), yielding the TßRIII-GFP expression plasmids. The plasmids were confirmed by DNA sequencing.

\section{Cell culture and transfection}

HeLa, L6 and MCF7 cells (Cell Resource Center, IBMS, CAMS/PUMC) were cultured in DMEM (Gibco) supplemented with $10 \%$ fetal bovine serum (Hyclone) at $37{ }^{\circ} \mathrm{C}$ in $5 \% \mathrm{CO}_{2} . \mathrm{R} 1 \mathrm{~B} /$ L17 cells were maintained in MEM containing 10\% FBS. Transfection was performed using Lipofectamine2000 (Invitrogen). Before transfection, cells were starved for $2 \mathrm{~h}$ in serum-free DMEM. Then, cells growing in a $35-\mathrm{mm}$ glass-bottom dish (Shengyou Biotechnology, China) were transfected with $0.25 \mu \mathrm{g}$ plasmids in the serum-free and phenol red-free DMEM. To increase the protein expression level, the cells were serum-starved and transfected with $0.5 \mu \mathrm{g}$ plasmids for the first $4 \mathrm{~h}$, washed, changed to DMEM medium with serum for another 2-10 h, serum-starved again in DMEM for 1-2 $\mathrm{h}$, then fixed and imaged in PBS buffer.

For the ligand stimulation experiments, the cells were treated with 200 pM TGF- $\beta 1$ (R\&D, USA) in DMEM for 15 min at $4{ }^{\circ} \mathrm{C}$ before fluorescence imaging. Then the cells were fixed in cold $4 \%$ paraformaldehyde/PBS solution for at least $15 \mathrm{~min}$ and washed 2 times. Finally, the cells were added with PBS buffer for fluorescence imaging.

\section{Single-molecule fluorescence imaging}

Single-molecule fluorescence imaging was performed with the objective-type TIRFM using an inverted Olympus IX71 microscope equipped with a total internal reflective fluorescence illuminator, a $100 \times / 1.45$ NA Plan Apochromat TIR objective and an electron-multiplying charge-coupled device (EMCCD) camera (Andor iXon DU-897D BV) [13]. GFP was excited at $488 \mathrm{~nm}$ by an argon laser (Melles Griot, Carlsbad, CA, USA) with the power of $5 \mathrm{~mW}$ measured after the laser passing through the objective. The collected fluorescent signals were passed through a filter HQ 525/50 (Chroma Technology), then directed to the EMCCD camera. The gain of the EMCCD camera was set at 300. Movies of 200-300 frames were acquired for each sample at a frame rate of $10 \mathrm{~Hz}$.

\section{Image analysis}

To analyze the single-molecule imaging of T $\beta R I$ and T $\beta$ RIII, the background fluorescence was firstly subtracted from the movie acquired from the fixed cells using the rolling ball method in NIH Image J software (Supplementary information, Figure S6). To select the regions of interest for bleaching analysis and receptor density counting, an imaging processing program written in Matlab (Mathworks Cor.) was developed based on the previously reported method with further improvements [13]. In brief, the first 
five frames of each movie were averaged. The averaged image was low-pass filtered with a 2-pixel cut-off and high-pass filtered with a 5-pixel cut-off. Then the images were applied a threshold to obtain regions of interest, i.e. diffraction-limited fluorescent spots. The threshold was set at 10 times SD measured in an area without cells (In our imaging condition, the threshold was set at 80 counts.). The pixels above the threshold in the image were considered as the regions of interests and $5 \times 5$ regions with the peaks as their centers were consecutively fitted by a 2-D Guassian function. The Guassian function is defined as

$$
\mathrm{I}=\operatorname{aexp}\left[-\frac{1}{2}\left[\left(\frac{x-b}{d}\right)^{2}+\left(\frac{y-c}{e}\right)^{2}\right]\right]+f
$$

where $a$ is the amplitude, $b$ and $c$ are the coordinates of the center of each fluorescent spot, $d$ and $e$ are standard deviations of the distribution in each direction, and $f$ is a constant term determined by background fluorescence, detector noise, and constant (noiseless) CCD offset [24]. We defined two parameters to determine whether the fitting result was a good Guassian fitting: roundness $\left(d^{2} /\right.$ $e^{2}$ ) and sharpness (the FWHM of a Guassian fitting). The regions (5 $\times 5$ pixel) were considered as diffraction-limited fluorescent spots; if the convergence of the fitting was reached, the roundness of the fitting ranged between 0.7 and 1.4, and the sharpness ranged between $2 / 3$ and 3 . These diffraction-limited fluorescent spots which fitted to the Guassian function well were selected for calculating the receptor densities. Time courses of the integrated fluorescence intensity of the regions were extracted for bleaching analysis. Traces with erratic behavior and ambiguities (about $30 \%$ of traces) were discarded [13].

\section{Acknowledgments}

This work was supported by the National Natural Science Foundation of China (90713024, 20821003, 30921004), the National Basic Research Program of China (2007CB935601, 2010CB833706) and the Chinese Academy of Sciences.

\section{References}

1 Massague J, Chen Y. Controlling TGF- $\beta$ signaling. Genes Dev 2000; 14:627-644.

2 Dijke PT, Hill CS. New insights into TGF- $\beta$-Smad signalling. Trends Biochem Sci 2004; 29:265-273.

3 Kang JS, Liu C, Derynck R. New regulatory mechanisms of TGF- $\beta$ receptor function. Trends Cell Biol 2009; 19:385-394.

4 Boyd FT, Cheifetz S, Andres J, Laiho M, Massagu J. Transforming growth factor-beta receptors and binding proteoglycans. J Cell Sci 1990; 13:131.

5 Chen RH, Derynck R. Homomeric interactions between type II transforming growth factor-beta receptors. J Biol Chem 1994; 269:22868-22874.

6 Henis YI, Moustakas A, Lin HY, Lodish HF. The types II and III transforming growth factor- $\beta$ receptors form homooligomers. J Cell Biol 1994; 126:139-154.

7 Gilboa L, Wells RG, Lodish HF, Henis YI. Oligomeric structure of type I and type II transforming growth factor- $\beta$ recep- tors: homodimers form in the ER and persist at the plasma membrane. J Cell Biol 1998; 140:767-777.

8 Shi Y, Massagu J. Mechanisms of TGF- $\beta$ signaling from cell membrane to the nucleus. Cell 2003; 113:685-700.

9 Wells RG, Yankelev H, Lin HY, Lodish HF. Biosynthesis of the Type I and Type II TGF- $\beta$ receptors. J Biol Chem 1997; 272:11444-11451.

10 Ulbrich MH, Isacoff EY. Subunit counting in membranebound proteins. Nat Meth 2007; 4:319-321.

$11 \mathrm{Ji} \mathrm{W}, \mathrm{Xu} \mathrm{P}, \mathrm{Li} \mathrm{Z}$, et al. Functional stoichiometry of the unitary calcium-release-activated calcium channel. Proc Natl Acad Sci USA 2008; 105:13668.

12 Kohout SC, Ulbrich MH, Bell SC, Isacoff EY. Subunit organization and functional transitions in Ci-VSP. Nat Struct Mol Biol 2008; 15:106-108.

13 Zhang W, Jiang Y, Wang Q, et al. Single-molecule imaging reveals transforming growth factor- $\beta$-induced type II receptor dimerization. Proc Natl Acad Sci USA 2009; 106:1567915683.

14 Xiao Z, Ma X, Jiang Y, et al. Single-molecule study of lateral mobility of epidermal growth factor receptor 2/HER2 on activation. J Phys Chem B 2008; 112:4140-4145.

15 Cochet C, Kashles O, Chambaz EM, et al. Demonstration of epidermal growth factor-induced receptor dimerization in living cells using a chemical covalent cross-linking agent. $J$ Biol Chem 1988; 263:3290-3295.

16 Dennler S, Itoh S, Vivien D, et al. Direct binding of Smad3 and Smad4 to critical TGF beta-inducible elements in the promoter of human plasminogen activator inhibitor-type 1 gene. Embo J 1998; 17:3091-3100.

17 Sun L, Wu G, Willson JK, et al. Expression of transforming growth factor beta type II receptor leads to reduced malignancy in human breast cancer MCF-7 cells. J Biol Chem 1994; 269:26449-26455.

18 Boyd FT, Massague J. Transforming growth factor-beta inhibition of epithelial cell proliferation linked to the expression of a 53-kDa membrane receptor. J Biol Chem 1989; 264:2272-2278.

19 Arteaga CL, Tandon AK, Von Hoff DD, Osborne CK. Transforming growth factor $\beta$ : potential autocrine growth inhibitor of estrogen receptor-negative human breast cancer cells. Cancer Res 1988; 48:3898-3904.

20 Massague J. TGF- $\beta$ signal transduction. Annu Rev Biochem 1998; 67:753-791.

21 Groppe J, Hinck CS, Samavarchi-Tehrani P, et al. Cooperative assembly of TGF- $\beta$ superfamily signaling complexes is mediated by two disparate mechanisms and distinct modes of receptor binding. Mol Cell 2008; 29:157-168.

22 Lopez-Casillas F, Wrana JL, Massagu J. Betaglycan presents ligand to the TGF- $\beta$ signaling receptor. Cell 1993; 73:14351444.

23 Schlessinger J. Cell signaling by receptor tyrosine kinases. Cell 2000; 103:211-225.

24 Yildiz A, Forkey JN, McKinney SA, et al. Myosin V walks hand-over-hand: single fluorophore imaging with 1.5-nm localization. Science 2003; 300:2061.

(Supplementary information is linked to the online version of the paper on Cell Research website.) 